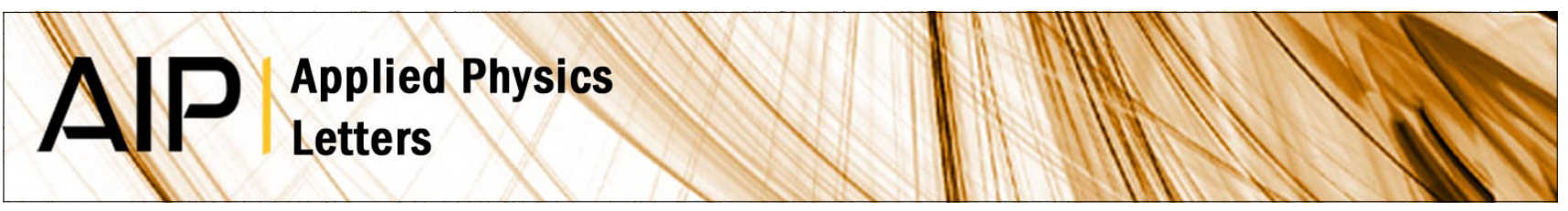

\title{
Electron beam induced current in InSb-InAs nanowire type-III heterostructures
}

C. Y. Chen, A. Shik, A. Pitanti, A. Tredicucci, D. Ercolani et al.

Citation: Appl. Phys. Lett. 101, 063116 (2012); doi: 10.1063/1.4745603

View online: http://dx.doi.org/10.1063/1.4745603

View Table of Contents: http://apl.aip.org/resource/1/APPLAB/v101/i6

Published by the American Institute of Physics.

\section{Related Articles}

Thermally driven unipolar and bipolar spin diode based on double quantum dots J. Appl. Phys. 112, 084324 (2012)

Temperature-dependent properties of semimetal graphite-ZnO Schottky diodes Appl. Phys. Lett. 101, 162106 (2012)

Millimeterwave Schottky diode on grapene monolayer via asymmetric metal contacts J. Appl. Phys. 112, 084302 (2012)

Electrical and microstructural analyses of $200 \mathrm{MeV}$ Ag14+ ion irradiated Ni/GaN Schottky barrier diode Appl. Phys. Lett. 101, 153508 (2012)

Theory of the suspended graphene varactor

Appl. Phys. Lett. 101, 153102 (2012)

\section{Additional information on Appl. Phys. Lett.}

Journal Homepage: http://apl.aip.org/

Journal Information: http://apl.aip.org/about/about_the_journal

Top downloads: http://apl.aip.org/features/most_downloaded

Information for Authors: http://apl.aip.org/authors

\section{ADVERTISEMENT}
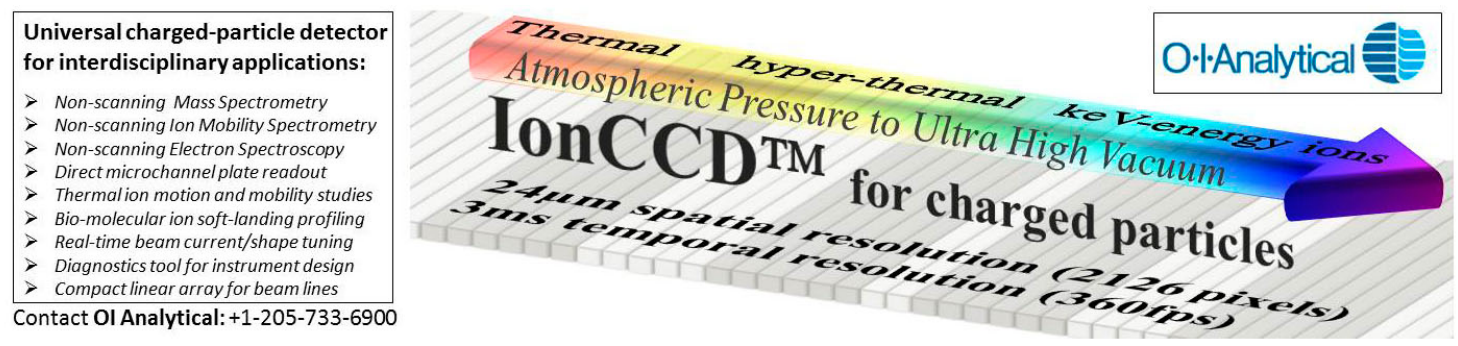


\title{
Electron beam induced current in InSb-InAs nanowire type-III heterostructures
}

\author{
C. Y. Chen, ${ }^{1, a)}$ A. Shik, ${ }^{1}$ A. Pitanti, ${ }^{2,3}$ A. Tredicucci, ${ }^{2}$ D. Ercolani, ${ }^{2}$ L. Sorba,${ }^{2}$ F. Beltram, ${ }^{2}$ \\ and H. E. Ruda ${ }^{1}$ \\ ${ }^{1}$ Centre for Advanced Nanotechnology, University of Toronto, 170 College Street, Toronto, Ontario M5S 3E3, \\ Canada \\ ${ }^{2}$ NEST, Scuola Normale Superiore and CNR-Istituto Nanoscienze, piazza San Silvestro 12, I-56127, Italy \\ ${ }^{3}$ Thomas J. Watson Sr. Laboratory of Applied Physics, California Institute of Technology, Pasadena, \\ California 91125, USA
}

(Received 6 June 2012; accepted 30 July 2012; published online 9 August 2012)

\begin{abstract}
InSb-InAs nanowire heterostructure diodes investigated by electron beam induced current (EBIC) demonstrate an unusual spatial profile where the sign of the EBIC signal changes in the vicinity of the heterointerface. A qualitative explanation confirmed by theoretical calculations is based on the specific band diagram of the structure representing a type-III heterojunction with an accumulation layer in InAs. The sign of the EBIC signal depends on the specific parameters of this layer. In the course of measurements, the diffusion length of holes in InAs and its temperature dependence are also determined. (C) 2012 American Institute of Physics. [http://dx.doi.org/10.1063/1.4745603]
\end{abstract}

Recent development in epitaxial growth of nanowires (NWs) allows accommodation for greater elastic strain than conventional planar structures, ${ }^{1}$ widening the choice of materials for growing defect-free heterostructures. One example is the InSb-InAs heterostructure with $\sim 7 \%$ in lattice mismatch where high-quality heterojunctions can be obtained only in NW form. ${ }^{2}$ The III-V semiconductor system InSb-InAs has the smallest bandgap with the highest electron mobility and presents a potential interest for future high speed electronic devices and far-infrared optoelectronics. 3,4 In this letter, we studied the diffusion of non-equilibrium carriers in an InSb-InAs NW heterostructure diode using the electron beam induced current (EBIC) technique.

The InSb-InAs heterostructure NWs were grown on an InAs(111)B substrate by chemical beam epitaxy through the $\mathrm{Au}$-assisted mechanism described in details in previous publication. ${ }^{5}$ The InAs part of the NW had a diameter $\sim 50 \mathrm{~nm}$ while the InSb segment was slightly thicker, due to the change in solubility of Au catalyst during growth. Separate NWs fabricated in identical technological conditions ${ }^{6}$ showed that both $\mathrm{InSb}$ and InAs have n-type conductivity with InAs having electron concentration of $3 \times 10^{17} \mathrm{~cm}^{-3}$ and InSb characterized by the resistivity $0.18 \Omega \mathrm{cm}$, which for typical mobilities in such NWs corresponds to the concentration $10^{16} \sim 10^{17} \mathrm{~cm}^{-3}$.

To fabricate the device measured in this letter, InSbInAs NWs were mechanically transferred from the growth substrate onto a $\mathrm{p}^{+}-\mathrm{Si}$ substrate capped with $100 \mathrm{~nm} \mathrm{SiO}_{2}$. An electron beam lithography system was used to locate the NWs and define contact electrodes. Prior to metal deposition, NWs were passivated with $\left(\mathrm{NH}_{4}\right)_{2} \mathrm{~S}_{\mathrm{x}}$ to secure a good ohmic contact between semiconductor and metal. Finally, electron beam evaporation was used to deposit $\mathrm{Ti}(10 \mathrm{~nm}) / \mathrm{Au}(130 \mathrm{~nm})$ contacts. A SEM image of the fabricated device is shown in Fig. 1(a). The InSb segment is the one closer to the lower electrode.

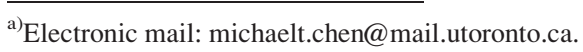

To investigate the transport of minority carriers and measure their diffusion length $L$, the technique of EBIC, characterized by the best spatial resolution among other methods, was selected. The electron beam created by SEM525 (Philips) operating at $10 \mathrm{kV}$ with the beam current $\sim 150 \mathrm{pA}$ and diameter $\sim 20 \mathrm{~nm}$, equipped with SEMICAPS 1000 image acquisition system, generated electron-hole pairs at a selected location of a NW. For EBIC measurements, the two electrodes shown in Fig. 1(a) were connected to a current preamplifier (SR570) with a gain of $1 \mathrm{nA} / \mathrm{V}$. The total capacitance of the measurement circuit was $\sim 200 \mathrm{pF}$. In the absence of applied voltage, EBIC has the same nature as the current in a photodiode being due to the separation of nonequilibrium carriers by the built-in field of the junction and to the diffusion delivery of minority carriers to the area of this field. To measure the temperature dependence of EBIC, a home-made liquid nitrogen cryostat was also incorporated into the SEM chamber.

The EBIC image of the InSb-InAs at $100 \mathrm{~K}$ is shown in Fig. 1(b) while Fig. 2 depicts the EBIC profile while

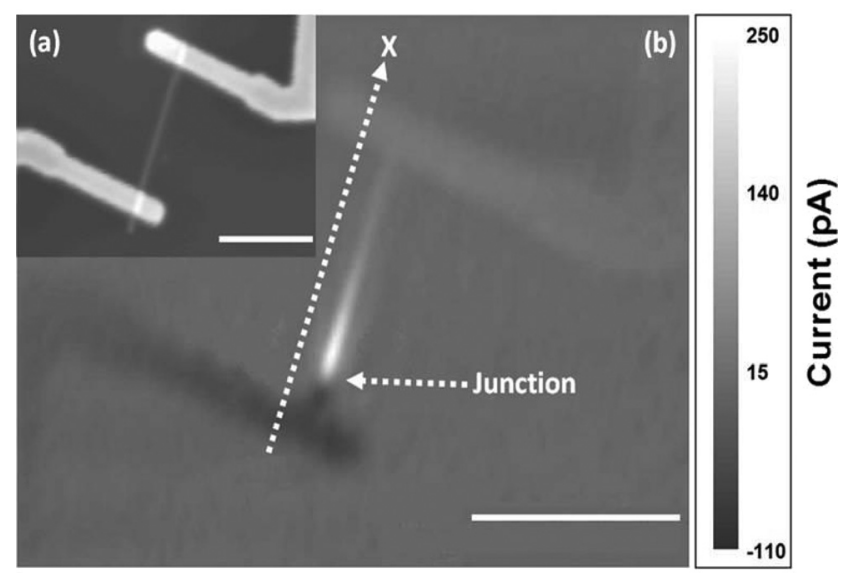

FIG. 1. SEM image of the InSb-InAs NW device (a) and its EBIC image at $100 \mathrm{~K}$ (b). The scale bar in both pictures is equal to $1 \mu \mathrm{m}$. The dashed coordinate axis shows the direction of scan resulting in the EBIC profile of Fig. 2. 


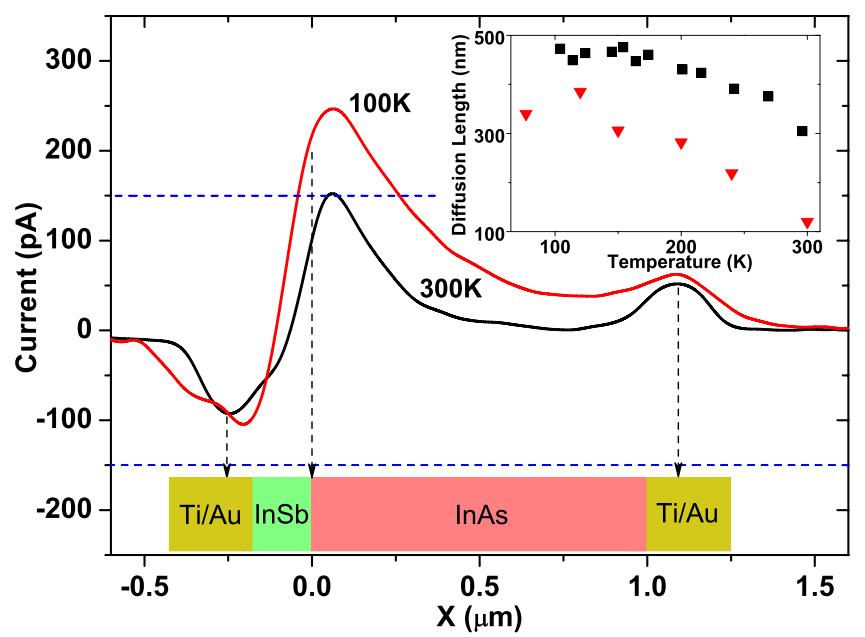

FIG. 2. Line scans of NW at $100 \mathrm{~K}$ and $300 \mathrm{~K}$. $x=0$ corresponds to the InSb-InAs interface. Horizontal dashed lines give for comparison the current in the electron beam. Inset: The diffusion length of holes in n-InAs vs temperature (square) compared to the similar data from Ref. 14 (triangle).

scanning along the NW (dashed line in Fig. 1(b)). In the image, the two contacts, top (bright-positive current) and bottom (dark-negative current), can be seen clearly. This contrast on the electrodes arises from direct absorption of the primary electron beam and their consequent current generation. Sometimes, it is called resistive EBIC (REBIC). ${ }^{7}$ In our setup, when electrons reach the current preamplifier via the lower electrode, contrast will be dark and bright for carriers reaching the amplifier through upper electrode. The REBIC current is only a fraction of the beam current which is shown by the horizontal dashed lines in Fig. 2. This fraction, collected by the upper electrode, is $30 \%-40 \%$ (for the lower electrode, the contact peak is partially overlapped with the signal from the NW and cannot be measured separately). It is in a good agreement with the results of direct Monte-Carlo simulation using the Casino v3.2 program, ${ }^{8}$ confirming that the contacts of our composition and thickness absorb most of the $10 \mathrm{kV}$ electron beam.

When the electron beam is focused on the NW, only a minor part of the beam current is absorbed by the NW. The large EBIC signal for $-0.2 \mu \mathrm{m}<x<0.9 \mu \mathrm{m}$, in some regions even exceeding the beam current, is explained only by the multiple electron-hole pair generation by high energy electrons, which is typically characterized by the quantum yield 100-1000 per primary electron. ${ }^{9}$ The most remarkable EBIC feature, seen directly in Fig. 2, is the change of signal sign in the vicinity of the junction, in contrast to EBIC in homo- and most hetero-junctions, where the properties of EBIC are described by the simple theory of a photodiode (see, e.g., Ref. 10). The latter predicts that the current has always the same sign, corresponding to the reverse current of the junction, and its dependence on the beam position is a singlemaximum curve with the width equal to the sum of the minority carrier diffusion lengths in $\mathrm{n}$ - and p-regions. In a double heterostructure, a non-monotonic EBIC profile was reported $^{11}$ but no sign change was observed. The unusual properties of the InSb-InAs NW heterojunction are connected with the specific band diagram of this system representing the so-called type-III broken-band alignment, with the conduction band of InAs lying below the valence band of

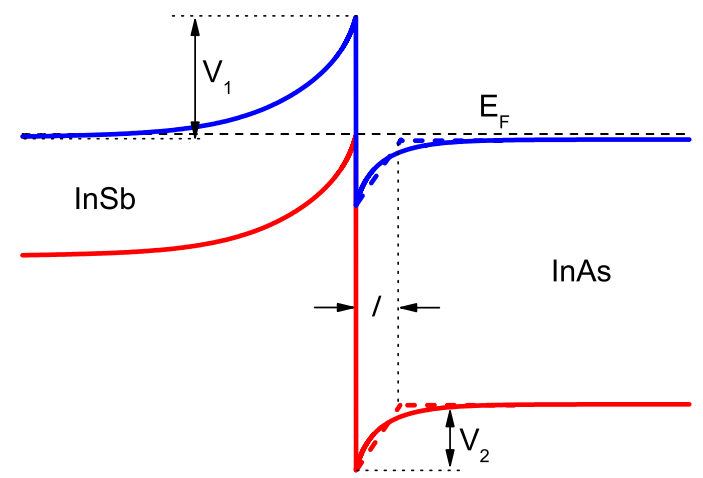

FIG. 3. Band diagram of the type-III heterojunction in the InSb-InAs system.

InSb. This rare type of band diagram, for lattice-matched heteropairs realized only in the InAs-GaSb system, ${ }^{12}$ is schematically shown in Fig. 3.

Theoretical analysis of the EBIC in our system should take into account that, contrary to the standard p-n junction, n-InAs with its anomalously large electron affinity contains an accumulation, rather than depletion, layer near the junction. The behavior of minority carriers (holes) in such layer is not so straightforward. In fact, it is seen from Fig. 3 that, on the one hand, InSb represents a potential well for nonequilibrium holes created in InAs and thus attracts these holes. On the other hand, the junction electric field prevents the holes from moving towards the interface, which requires them to overcome the potential barrier $V_{2}$, as indicated in Fig. 3. To elucidate the dominating direction of hole motion and predict the sign of EBIC, we must then solve the driftdiffusion equation for non-equilibrium holes in InAs created by the electron beam at some point $x=x_{0}\left(x_{0}>0\right)$. We assume that the space charge region in InAs has the width $l$ $\left(l<x_{0}\right)$ and the average contact electric field in it is $E$, so that $e E l=V_{2}$. In the region, $0<x<l$, the distribution of nonequilibrium holes $p(x)$ can be found by solving the continuity equation $-p^{\prime \prime}+\frac{e E}{k T} p^{\prime}+\frac{p}{L^{2}}=0 \quad(L$ is the diffusion length for holes) which has, at $l \ll L$, the general solution $p(x)$ $=A \exp \left(\frac{e E x}{k T}\right)+B \exp \left(-\frac{k T x}{e E L^{2}}\right)$. For $x>l$, the drift term is absent and the solution is $p(x)=C \exp \left(\frac{x}{L}\right)+D \exp \left(-\frac{x}{L}\right)$ at $l<x<x_{0}$ and $p(x)=F \exp \left(-\frac{x}{L}\right)$ at $x>x_{0}$. The coefficients $A, B, C, D, F$ are determined from the following five conditions: (1) $p(0)=0$ (deep potential well), (2 and 3 ) continuity of $p(x)$ at $x=l$ and $x=x_{0},(4)$ continuity of current at $x=l$, (5) continuity of current at $x=x_{0}$ where the electron beam creates $G$ electron-hole pairs per unit area per second: $C \exp \left(\frac{x_{0}}{L}\right)+(F-D) \exp \left(-\frac{x_{0}}{L}\right)=\frac{G L}{D_{p}}$ with $D_{p}$ being the diffusion coefficient of holes.

Using these conditions, we find the coefficients in the concentration profile and with their help determine the current density in the external circuit, which is computed as the difference of hole fluxes right and left from the plane $x=x_{0}$,

$$
\begin{aligned}
j & =-\frac{e D_{p}}{L}\left[(D+F) \exp \left(-\frac{x_{0}}{L}\right)-C \exp \left(\frac{x_{0}}{L}\right)\right] \\
& =-e G \frac{\exp \left(\frac{V_{2}}{k T}\right)-1-\frac{e E L}{k T}}{\exp \left(\frac{V_{2}}{k T}\right)-1+\frac{e E L}{k T}} \exp \left(-\frac{2 x_{0}}{L}\right) .
\end{aligned}
$$

It is seen that the sign of the current depends on the factor $A \equiv \exp \left(\frac{V_{2}}{k T}\right)-1-\frac{e E L}{k T}$. For our case, the self- 
consistent calculations of the accumulation layer in InAs, using the theoretical formulae of Ref. 13 , give $V_{2} \approx 0.06 \mathrm{eV}$ and $E \approx 75 \mathrm{kV} / \mathrm{cm}$. In this case, the factor $A$ at room temperature is negative for $L>30 \mathrm{~nm}$, which is shown below to be well met in our structures (see the insert in Fig. 2, more on this later). It means that non-equilibrium holes in InAs move towards InSb corresponding to the positive sign of EBIC. The same sign of current is realized when the electron beam bombards the upper contact directly.

Electron-hole pairs generated by the electron beam in InSb are separated in the depletion layer, exactly as in an ordinary $\mathrm{p}-\mathrm{n}$ junction. In our case this corresponds to the flux of non-equilibrium holes from InSb into InAs, that is, in the opposite direction for pair generation in InAs, which corresponds to the negative current in Fig. 2. The same direction of current is realized when the electrons bombard the lower contact and leave into the external circuit. Thus the discussed physical processes well describe the picture shown in Fig. 2 and characterized by different signs of EBIC at both sides of the InSb-InAs heterojunction.

We see from Eq. (1) that moving the beam away from the junction results in the exponential drop of EBIC with the characteristic length determined by $L$, similar to an ordinary p-n junction (described, e.g., in Ref. 10). This allows us to determine the value of $L$ by measuring the spatial variation of the EBIC signal right of the main maximum in Fig. 2 (in the InAs segment) but not too close to the second maximum (where it is influenced by the contact), namely, in the distance interval $0.1-0.7 \mu \mathrm{m}$.

The same procedure was repeated at different temperatures and resulted in the temperature dependence of $L$ presented in the inset of Fig. 2. It has comparable value and similar temperature dependence in the interval 100-300 K with the diffusion length in InAs observed in Ref. 14 (to be more precise, Dupuy et al. ${ }^{14}$ measured the ambipolar diffu- sion length which in $n$-type samples must be close to the hole diffusion length $L$ ). The temperature dependence of $L$ can be attributed to the drop in mobility caused by phonon scattering. For phonon scattering, the mobility in different InAs structures must have a universal value, varying in the interval $100-400 \mathrm{~cm}^{2} / \mathrm{V} \mathrm{s} .{ }^{15}$ This allows us to estimate the hole lifetime having the order of $4 \times 10^{-10} \mathrm{~s}$ in our samples and $5 \times 10^{-11} \mathrm{~s}$ in Dupuy et al. ${ }^{14}$

Authors C.Y.C., A.S., and H.E.R. gratefully acknowledge support for this work from NSERC, CIPI, CSA, and OCE. A.P. acknowledges funding from European Union through Marie Curie Actions under REA grant agreement 298861 (NEMO).

${ }^{1}$ K. L. Kavanagh, Semicond. Sci. Technol. 25, 024006 (2010).

${ }^{2}$ P. Caroff, M. M. Messing, B. M. Borg, K. A. Dick, K. Deppert, and L.-E. Wernersson, Nanotechnology 20, 495606 (2009).

${ }^{3}$ P. Caroff, J. B. Wagner, K. A. Dick, H. A. Nilsson, M. Jeppsson, K. Deppert, L. Samuelson, L. R. Wallenberg, and L.-E. Wernersson, Small 4, 878 (2008).

${ }^{4}$ H. A. Nilsson, P. Caroff, C. Thelander, E. Lind, O. Karlstrom, and L.-E. Wernersson, Appl. Phys. Lett. 96, 153505 (2010).

${ }^{5}$ D. Ercolani, F. Rossi, A. Li, S. Roddaro, V. Grillo, G. Salviati, F. Beltram, and L. Sorba, Nanotechnology 20, 505605 (2009).

${ }^{6}$ A. Pitanti, D. Ercolani, L. Sorba, S. Roddaro, F. Beltram, L. Nasi, G. Salviati, and A. Tredicucci, Phys. Rev. X 1, 011006 (2011).

${ }^{7}$ J. D. Russell and C. Leach, Acta Mater. 17, 6227 (1998).

${ }^{8}$ H. Demers, N. Poirier-Demers, A. R. Couture, D. Joly, M. Guilmain, N. D. Jonge, and D. Drouin, Scanning 33, 135 (2011).

${ }^{9}$ H. J. Leamy, J. Appl. Phys. 53, R51 (1982).

${ }^{10}$ D. B. Holt and D.C. Joy, SEM Microcharacterization of Semiconductors (Academic, London, 1989), Chap. 6, pp. 241-296.

${ }^{11}$ A. Gustafsson, M. T. Björk, and L. Samuelson, Nanotechnology 18, 205306 (2007).

${ }^{12}$ G. A. Sai-Halasz, R. Tsu, and L. Esaki, Appl. Phys. Lett. 30, 651 (1977).

${ }^{13}$ A. Shik and M. Singh, Phys. Status Solidi A 168, 195 (1998).

${ }^{14}$ E. Dupuy, D. Morris, N. Pauc, V. Aimez, M. Gendry, and D. Drouin, Appl. Phys. Lett. 94, 022113 (2009).

${ }^{15}$ O. Madelung, Semiconductors: Data Handbook, 3rd ed. (Springer, Berlin, 2004). 